\title{
Grab it! Biased attention in functional hand and tool space
}

\author{
Catherine L. REED \\ University of Denver, Denver, Colorado \\ and Claremont McKenna College, Claremont, California \\ AND \\ Ryan Betz, John P. Garza, ANd RalPh J. Roberts, JR. \\ University of Denver, Denver, Colorado
}

\begin{abstract}
This study explored whether functional properties of the hand and tools influence the allocation of spatial attention. In four experiments that used a visual-orienting paradigm with predictable lateral cues, hands or tools were placed near potential target locations. Results showed that targets appearing in the hand's grasping space (i.e., near the palm) and the rake's raking space (i.e., near the prongs) produced faster responses than did targets appearing to the back of the hand, to the back of the rake, or near the forearm. Validity effects were found regardless of condition in all experiments, but they did not interact with the target-in-grasping/raking-space bias. Thus, the topology of the facilitated space around the hand is, in part, defined by the hand's grasping function and can be flexibly extended by functional experience using a tool. These findings are consistent with the operation of bimodal neurons, and this embodied component is incorporated into a neurally based model of spatial attention.
\end{abstract}

Humans have evolved to perform functional and adaptive actions in the world. We must respond quickly and effectively when an enemy throws a rock or when a friend tosses an apple at us. In these situations, the current location of our limbs - and especially our hands - influences the speed and success with which we can either knock the rock away or grab the apple. These common examples emphasize the dynamic nature of the environment and the need for our spatial attention system to effectively coordinate visual and bodily information. Thus, visual spatial attention should be influenced by the current position of the hand and the specific functions it performs.

Spatial attention refers to the cognitive process through which certain visual stimuli are selected to the exclusion of other stimuli on the basis of their spatial location (e.g., Vecera \& Rizzo, 2003). One of the primary functions of spatial attention is to select objects and locations in space that are functionally relevant to what we are doing now or are about to do (Tipper, 2004). Spatial attention may amplify signals associated with salient regions of space and, thereby, improve perceptual processing (Braun, Koch, \& Davis, 2001; Pashler, 1998; Posner \& Cohen, 1984). Attention can be controlled in different ways. It can be directed from bottom-up inputs such as visual cues, and it can be directed on the basis of top-down sources such as motivation and goals. It may also be directed on the basis of inputs from the body. The current orientation of our bodies and, specifically, the positions of our hands change the salience of space by providing anchors or ref- erences for upcoming actions. However, few studies have examined how the actions of our hands and functional capabilities affect the distribution of spatial attention. In this article, we argue that the functional capabilities of our hands and our functional experiences with tools facilitate processing in the space relevant for upcoming actions.

We propose that theories of attention must include an embodied component that addresses how our bodies help shape the distribution of attention in space and how visual events are processed as a result (Reed, Garza, \& Roberts, 2007; Reed, Grubb, \& Steele, 2006). The current configuration of our body parts constrains our actions at any moment. As a result, body part location should influence where spatial attention is allocated across visual space. Several studies in neurologically intact populations have documented that attention and lateralized visual target detection are biased by trunk orientation both when one is standing and when one is walking (Grubb \& Reed, 2002; Grubb, Reed, Bate, Garza, \& Roberts, 2008; HasselbachHeitzeg \& Reuter-Lorenz, 2002).

In addition to the trunk, hand location also affects processing in regions of space in which functional actions can be performed - namely, peripersonal space. Researchers have distinguished among space on the body (personal space), space near the body (peripersonal), and space far from the body (extrapersonal) (e.g., Previc, 1998; Rizzolatti, Gentilucci, \& Matelli, 1985). The importance of objects in peripersonal space and, especially, in perihand space is that one can grasp them. They are candidates

C. L. Reed, clreed@cmc.edu 
for action or potential vehicles for performing important functions. As a result, both the presence of the hand near an object and the way that we use our hands may change the way that the object is represented and the way that attention is distributed to that region of space. In this study, we investigate whether attention to space near the hand is differentially influenced by the functional capabilities of the hand and our functional experience with tools.

Neurophysiological, neuropsychological, and psychological studies suggest that attention might be influenced differentially by visual targets appearing near the hand, in part because of bimodal neurons that respond to tactile stimulation on the hand, as well as to visual stimuli located near the hand (Graziano \& Gross, 1994, 1995). Consequently, a visual target near the hand is represented not only by visual neurons representing visual targets, but also by bimodal neurons that are specific to the region on and near the hand. As a result, visual targets appearing in space near the hand may be represented more strongly than targets appearing far from the hand. The locations of these bimodal neuron populations in the ventral intraparietal sulcus, polysensory zone, and putamen form a multimodal neural network that is situated to prioritize space for upcoming action (Graziano \& Cooke, 2006). If bimodal neurons serve the purpose of coordinating visual and tactile-motor systems when interaction with the world is required, the distribution of attention may be different for target locations around the hand. In other words, this bimodal neural network may provide information relevant to future actions and bias the attention system to attend to space near the hand. An open question is whether functional experience using the hand changes the relative contribution of this additional processing to regions that correspond with the grasping space of the hand, relative to less functional regions around the hand.

Consistent with the idea that these bimodal representations of space support functional action, research with primates has demonstrated that the extent of these peripersonal, bimodal representations is malleable. Iriki, Tanaka, and Iwamura (1996) found that if a macaque monkey used a rake to manipulate objects, the receptive fields of bimodal cells that previously encoded the location of objects relative to the hand expanded to code for objects relative to the rake. Both functionally and neurally, the rake became an extension of the monkeys' hands. Thus, the receptive fields of bimodal neurons in monkeys can be modified by functional experience.

Similar plasticity of functional space has been indicated from brain-damaged patients. Farné and Ládavas (2000) reported that spatial representations of peripersonal space in tactile extinction patients can be modified through functional interactions with objects, such as tools. They found that visual stimuli near the ipsilesional hand extinguished tactile stimuli on the contralesional hand but that visual stimuli at greater distances did not. However, after patients had used a tool to manipulate objects outside of their reach, visual stimuli presented near the tool held in the ipsilesional hand extinguished tactile stimuli on the contralesional hand. The representation of peripersonal space appeared to have expanded to incorporate the tool's extent.
This suggests that representations of multimodal peripersonal space can be modified by performing goal-directed movements through a physical extension of the hand.

Recently, research in neurologically intact humans has demonstrated that the allocation of spatial attention is influenced by hand location. Using a purely visual, predictive covert-orienting task, Reed et al. (2006) demonstrated that passive hand position influenced spatial attention, even though the hand was completely irrelevant to the task. Participants held one hand next to one of two lateralized target locations. Hand location changed attention to the space near the hand: The participants were faster to detect targets appearing next to the hand. However, hand location did not influence cue effectiveness, as measured by the size or presence of the validity effect.

\section{The Present Study}

In this study, we explored whether the way we use our hands and tools influences the distribution of attention in perihand, peripersonal, and extrapersonal space. In four experiments, we investigated whether the distribution of spatial attention would be influenced by the hand's functions and, if so, whether the functional topology was malleable so as to extend beyond the hand's reaching space, using grasped tools. Extinction research from neurological patients (e.g., Ládavas, Pellegrino, Farné, \& Zeloni, 1998), single-cell recording research from primates (e.g., Iriki et al., 1996), and behavioral cross-modal research from neurologically intact humans (e.g., Holmes, Calvert, \& Spence, 2006; Maravita, Spence, Kennett, \& Driver, 2002) suggest that it may. Using a predictive covertorienting paradigm (Posner, Walker, Friedrich, \& Rafal, 1987; Reed et al., 2006), we examined whether hand and tool function would affect the distribution of spatial attention. Would the ways in which we use our hands and tools bias spatial attention in perihand and peripersonal space, and could this biased allocation be extended via tool use to include extrapersonal space? In Experiments 1 and 2, we manipulated the type of interaction that could be performed by the hand while the hand's distance from the target was held constant. In Experiment 1, we examined the detection of targets that the hand could either grasp or hit. In Experiment 2, we examined the extent to which the biased allocation of attention was specific to the space around the hand. In Experiments 3 and 4, we investigated whether this functional space could be flexibly extended outside of peripersonal space via the use of a tool. Our primary hypothesis was that the functional capabilities of the hand in peripersonal space would change the spatial distribution of attention.

\section{EXPERIMENT 1 Functional Topology of Attentional Bias}

To the human, the importance of objects in peripersonal space is that you can grasp them - and they can grasp you! Either you want to grab them and work with them, or you want to hit them, avoid them, or push them away. As a result, the presence of the hand near an object changes the functional implications of the object and, potentially, 
the need to attend to that object. In Experiment 1, we used a predictive covert-orienting paradigm to investigate whether hand function would influence spatial bias for selective attention. Objects near the body are candidates for action. That action can be grasping or avoidance (e.g., Cooke \& Graziano, 2004). Correspondingly, the visual receptive fields of primate bimodal neurons around the hands are often mapped to include an equal proportion of space around the hand that can be relevant for both types of actions (e.g., Graziano \& Gross, 1995). Primates may have less of a bias for objects in front of the palm. However, in humans, objects in front of the palm are more typically acted on than are ones behind the hand. Alternatively, we more often use the palm of our hand to interact with objects than the back of our hand. This suggests that there may be a bias for humans to attend more to objects in front of the palm, as a result of functional experience in interacting with objects.

In this experiment, we determined whether effects of hand position are specific to the manipulation capabilities of the hand. The position of an object relative to the limb affects how we can interact with it. According to Graziano and Gross (1995) and others (Berti \& Frassinetti, 2000; Vaishnavi, Calhoun, \& Chatterjee, 1999), body-partcentered representations serve to facilitate the interaction between the body part and nearby objects. The kinds of actions one needs to perform to interact with an object in front of the palm are different from those required for an object behind the back of the hand. For example, we can immediately grasp an object that is in front of our palm but cannot grasp an object that is the same distance from the back of our hand without moving the hand first.

We compared two conditions that varied in terms of whether targets appear in the hand's grasping space (i.e., near the palm) or back-hand space. In the palm condition, the hand was placed to the outside of the target position, so that the target appeared on the palm side of the hand. In the back-hand condition, the hand was placed to the inside of the target position, so that the target appeared on the back side of the hand. If the biased space around the hand is equally distributed around the hand, we should find facilitated target detection for both the palm (i.e., grasp) and back-hand conditions. However, if the more extensive experience of grasping objects changes the distribution of spatial attention, we should find facilitated target detection for grasp conditions only.

Experiment 1 also explored the effects of hand function on different inputs affecting the allocation of attention. Do hand position and the peripheral visual cues act independently or combine to influence attention? In other words, the distribution of attention can be affected by hand position, the peripheral cue, or both. In this paradigm, cues and target boxes appeared on the left and right sides of fixation; targets appeared within the boxes. Our purpose was to demonstrate lateralized response differences corresponding to the location of a single hand. To do this, participants either placed the right hand next to the right target box or placed the left hand next to the left target box. If hand function biased space near the hand, a threeway interaction of hand position (grasp, back), hand side (left, right), and target side (left, right) would be expected that did not interact with cue validity (i.e., all conditions would show equivalent validity effects). Thus, evidence that both hand presence and hand function contribute to facilitated processing would be that targets appearing on the same side as the hand are detected more quickly than targets appearing away from the hand or to the back of the hand. Alternatively, if both hand function and peripheral cues affected attention, there should be a four-way interaction of validity with hand function, hand side, and target side.

\section{Method}

Participants. Twenty-seven right-handed undergraduates (16 female, mean age $=20.10$ years) from the University of Denver participated for extra credit.

Procedure. The participants performed a standard covert attention task (see Figure 1; Posner et al., 1987). Stimuli were presented on a 17-in. monitor using E-Prime 1.0 software (Psychological Software Tools, Pittsburgh, PA). The participants were presented with a central cross $\left(2^{\circ}\right)$, flanked by two empty squares $\left(2^{\circ}\right)$ located $5^{\circ}$ to

\section{Progression of Trials in Covert-Orienting Paradigm}

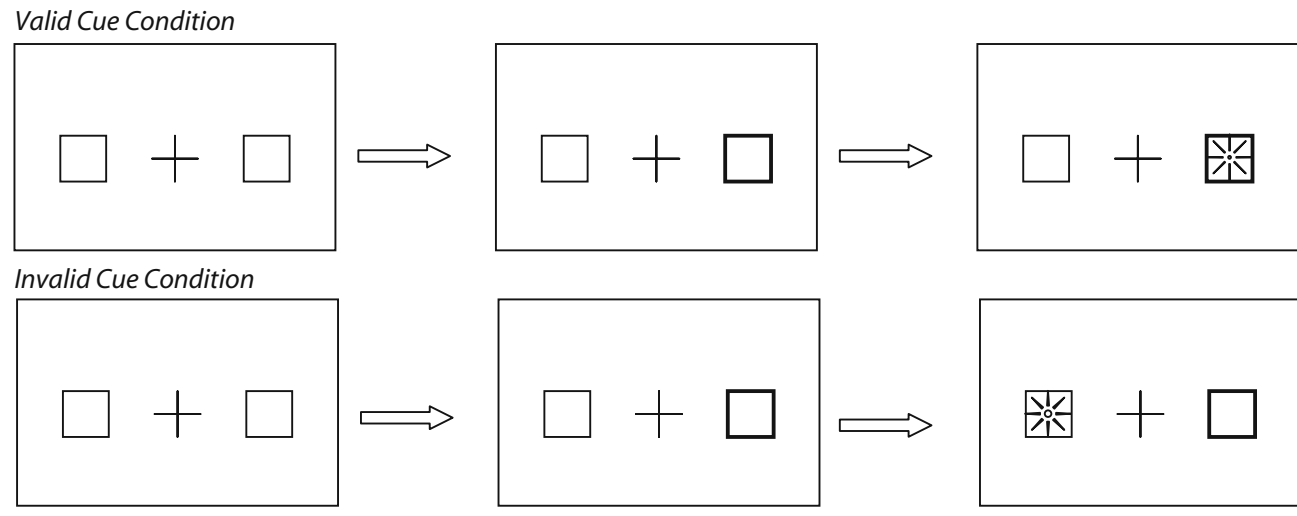

Figure 1. Progression of trials in the visual covert-orienting task. Each trial begins with a fixation cross between two boxes. Attention is validly cued to one of the box locations by a brightening of one of the boxes. For valid trials, the target appears within the cued box. For invalid trials, the target appears in the opposite box. 

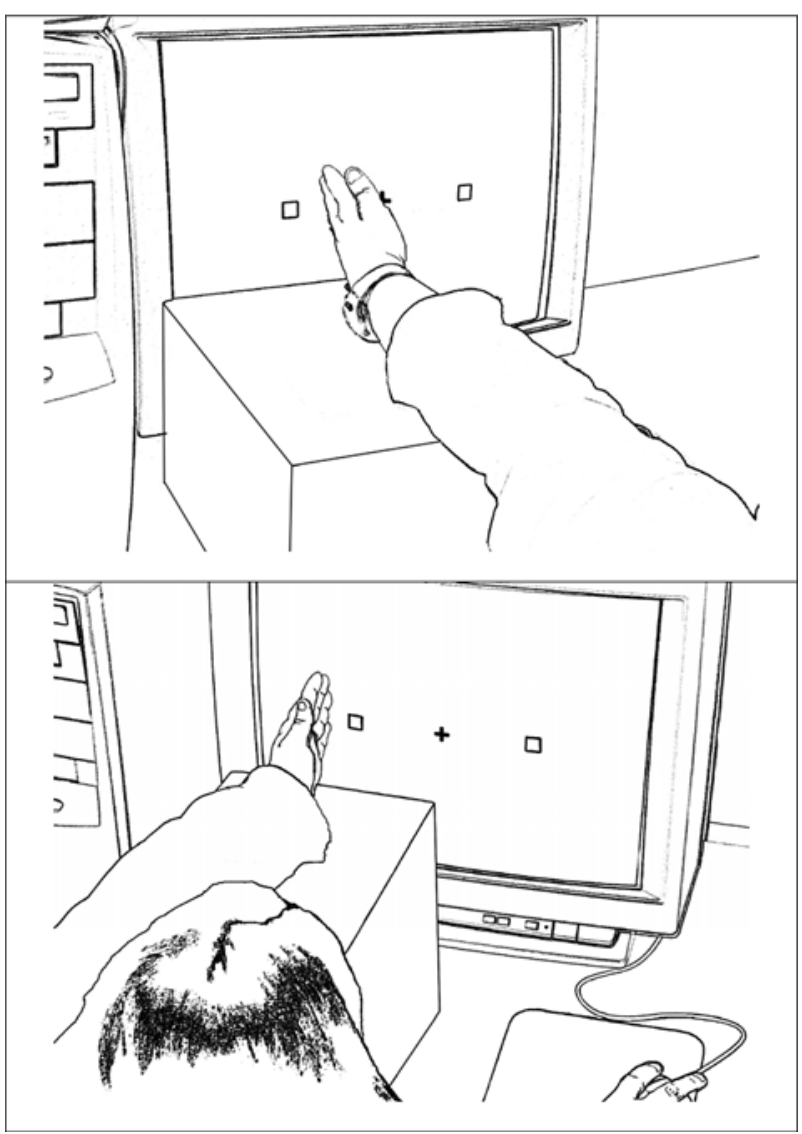

Figure 2. Experimental conditions for Experiment 1. In the palm conditions, the hand was held so that the target box was in front of the hand. In the back-hand conditions, the hand was held so that the target box was in back of the hand. In both conditions, the target box was the same distance from the hand.

either side. On each trial, the participants were instructed to fixate on the cross. Between 1,500 and 3,000 msec after the beginning of the trial, one square's border darkened. On valid trials, the target (i.e., a solid black dot; $1.8^{\circ}$ ) appeared within the cued square. On invalid trials, the target appeared in the square on the opposite side of the fixation cross. In addition, during catch trials, one square was cued, but no target appeared. Within each condition, $70 \%$ of the trials were validly cued, $20 \%$ were invalidly cued, and $10 \%$ were catch trials. For noncatch trials, the target appeared $200 \mathrm{msec}$ later. The participants were instructed to respond as quickly and accurately as possible to the target by pressing the mouse button on the table.

The comparison of performance in two experimental conditions indicated whether the functional properties of the hand influenced spatial attention: The side of the hand on which the target appeared was manipulated (see Figure 2) in the palm and back-hand conditions. For example, in the palm conditions for the left hand, the participants placed their left hand next to the outer edge of the left square with their palm facing the square; they responded by pressing a button with their opposite hand. In the back-hand conditions, the participants placed their left hand next to the inner edge of the left square with the back of their hand directed toward the square; the participants responded with their opposite hand. In both conditions, the participants held their hands so that their fingertips touched the computer screen and the thumb was directed up in a relaxed grasping position (Figure 2). In both conditions, the fixation and the target/ target box were clearly visible without eye movement. The arm was supported comfortably by a brace, and the experimenter made sure that the shoulder was relaxed. There were two blocks of trials for each condition (i.e., palm left, palm right, back left, back right) for a total of eight blocks of 50 trials each. Block order was randomized.

\section{Results and Discussion}

For each participant, mean response times (RTs) to targets were calculated for each side, cuing condition, and hand condition. To eliminate anticipation and inattention errors, trials on which the participants responded incorrectly or outside a time window from 200 to $900 \mathrm{msec}$ after the target appeared were excluded from the mean calculation. Two participants responded in excess of $25 \%$ of the catch trials and were excluded from the analyses. Alpha was set at the .05 level for this and all the subsequent experiments.

To determine whether hand function affected spatial attention, a $2 \times 2 \times 2 \times 2$ repeated measures ANOVA was conducted with factors of hand position (palm, back), hand side (left, right), target side (left, right), and validity (valid, invalid). Confirming the basic paradigm, valid trials $(M=341.92, S E=8.54)$ were responded to more quickly than were invalid trials $(M=370.76, S E=11.56)$ [main effect of validity: $F(1,24)=24.93, M S_{\mathrm{e}}=3,335.47$, $p<.0001]$. Most important, performance was moderated by the hand's position relative to the target. Targets appearing next to the hand on the palm side were detected relatively more quickly than were targets appearing next to the hand but to the back of the hand [Figure 3; hand position $\times$ hand side $\times$ target side interaction: $F(1,24)=$ 7.38, $\left.M S_{\mathrm{e}}=893.57, p=.012\right]$. To assess the differential effects of hand position, separate hand side $\times$ target side ANOVAs for palm and back-hand positions showed that this interaction between hand position and target side occurred only for the palm conditions $[F(1,24)=$ 7.25, $\left.M S_{\mathrm{e}}=396.86, p=.013\right]$ and not for the back-hand conditions $\left[F(1,24)=2.10, M S_{\mathrm{e}}=1,787.52, p>.16\right]$. However, this hand position facilitation effect occurred for both validly and invalidly cued trials: The four-way interaction that included cue validity was not significant $[F(1,24)<1$, n.s.]. The hand space in which the target occurred did not differentially influence the lateralized cue effect. No other main effect or interaction approached significance $(p>.09)$.

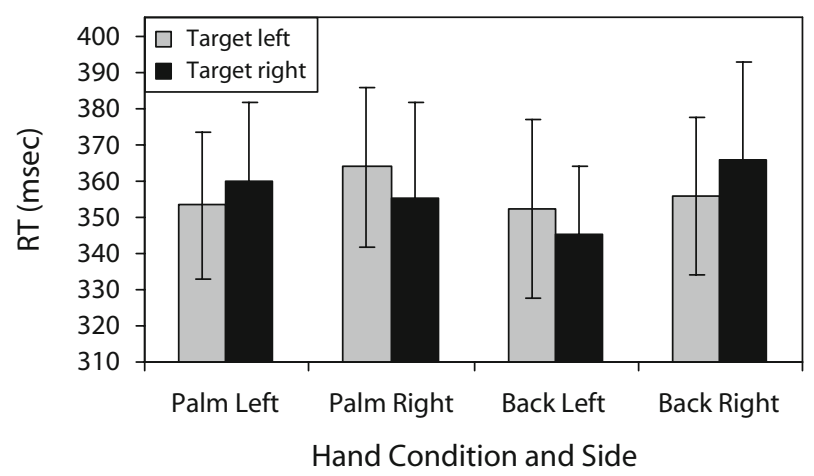

Figure 3. Results of Experiment 1. The results indicate a threeway interaction between hand position, hand side, and target side. They show facilitated response times (RTs) for targets appearing on the same side as the hand, but only when they appeared by the palm in grasping space. Error bars indicate $95 \%$ within-subjects confidence intervals. 
In sum, targets appearing in the grasping space of the hand (i.e., palm side of the hand) were detected more quickly than when they appeared by the back side of the hand, at an equidistant location. This finding suggests that our experience and functional use of our hands lead to greater bias for space near our hands. The hand's grasping function, rather than its avoidance or hitting function, appears to facilitate the further processing of objects.

It is important to note that the validity effect was not influenced by either the palm or the back-hand condition. In the back-hand condition, the hand was placed between the fixation and the target box. This hand location might have potentially blocked attentional processing of the cued target box locations (i.e., the finding of validity effects in the back-hand conditions), but this was not the case. The data revealed no differential effects of validity between the two hand conditions and revealed only effects of target detection.

Thus, these results support previous findings that space near the hand is biased for attention even if the hand is not directly involved in the task, either in terms of task demands (i.e., the task was purely visual) or responses (i.e., a different hand performed the response). The results of Experiment 1 extend our understanding of spatial attention by documenting how spatial attention may be biased for space around the hand, or perihand space. This attention bias for perihand space appears to be asymmetric, with priority given to targets appearing in grasping space.

\section{EXPERIMENT 2 Functional Specificity of Grasping Space}

In Experiment 2, we investigated whether attentional bias is specific to grasping space near the hand. In other words, is the bias greater in the space immediately next to the palm than in the more general hand/arm action space extending down the forearm? Both of these regions have been documented in primates to have receptive fields that are sensitive to visual and tactile stimulation, with some neurons being more hand centered and others more arm centered (e.g., Graziano, 2001; Graziano, Taylor, Cooke, \& Moore, 2005). In this experiment, we compared the detection of stimuli appearing next to the palm/hand or next to the forearm, using the same predictive covert-orienting paradigm as in Experiment 1. The participants placed either their hand or their forearm next to the outside of the left and right target boxes. If the attention bias is directed to arm-centered space or is more generally action centered, we should find bias effects in both conditions. However, if the functional experience of grasping differentially biases attention in grasping space immediately near the palm, we should find stronger effects in the hand condition.

\section{Method}

Participants. Thirty-four right-handed undergraduates (29 female, mean age $=19.93$ years) from the University of Denver participated for extra credit in psychology courses.

Procedure. The procedure was the same as that described in Experiment 1, except for the experimental conditions. This experiment had two conditions. In the hand condition, which was the same as the palm condition in Experiment 1, the hand was held next to the target

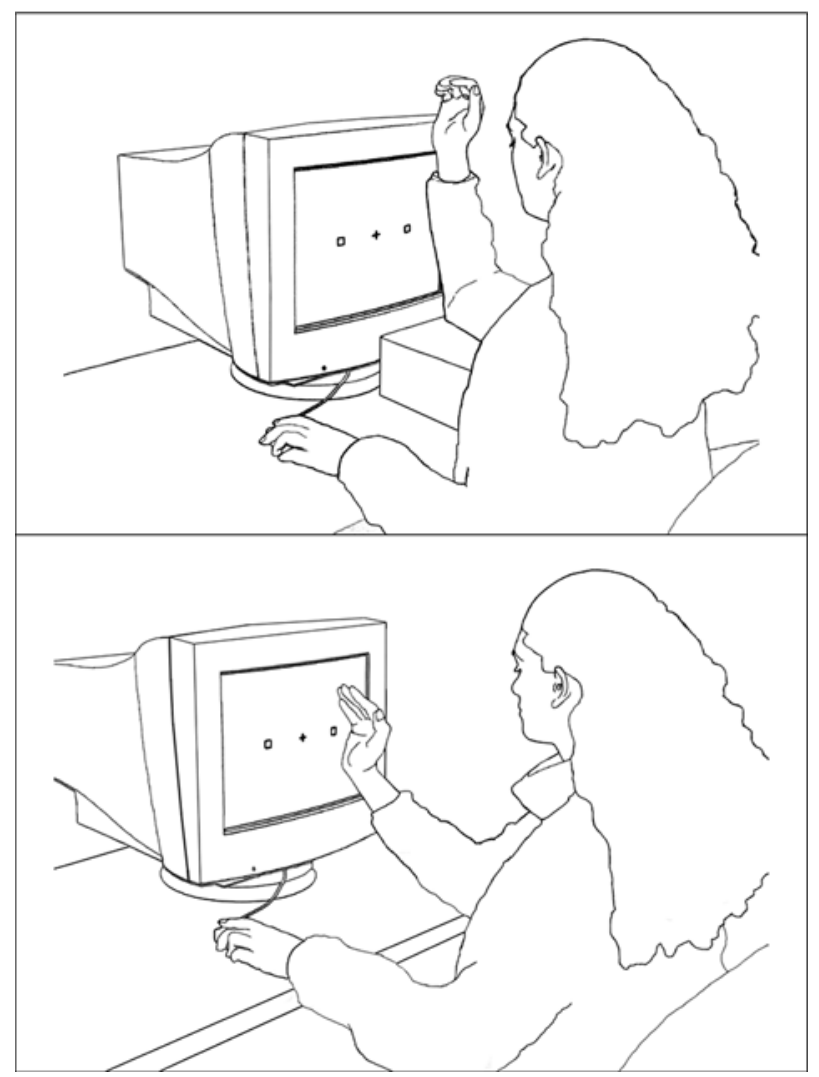

Figure 4. Experimental conditions for Experiment 2. For the palm conditions, the hand is held so that the target box is in front of the hand. For the forearm conditions, the middle of the forearm is held next to the target box in front of the arm.

box and the target appeared near the palm. The forearm condition required the participants to hold their forearm against the computer screen so that the target appeared next to the middle of the forearm (see Figure 4).

\section{Results and Discussion}

The data were processed in the same manner as in Experiment 1 . Four participants responded in excess of $25 \%$ of the catch trials and were excluded from the analyses.

To determine whether arm part affected attention, a $2 \times 2 \times 2 \times 2$ repeated measures ANOVA was conducted with the factors of arm part (hand, forearm), arm side (left, right), target side (left, right), and validity (valid, invalid). Confirming the basic paradigm $(M=361.74, S E=7.20)$, valid trials were responded to more quickly than invalid trials $(M=394.80, S E=8.15)$ [main effect of validity: $\left.F(1,28)=71.64, M S_{\mathrm{e}}=49,549.28, p<.0001\right]$. For both the hand and the forearm, the side of the arm influenced target detection time [Figure 5; arm side $\times$ target side interaction: $\left.F(1,28)=7.18, M S_{\mathrm{e}}=10,634.05, p=.012\right]$. Similar patterns of facilitation might suggest a gradient of facilitation that is stronger for the hand than for the forearm region, but there was no three-way interaction of arm part, arm side, and target side. Furthermore, separate arm side $\times$ target side $\times$ validity ANOVAs revealed that only the hand condition produced a significant interaction $\left[F(1,28)=5.28, M S_{\mathrm{e}}=506.01, p=.029\right]$; the forearm 


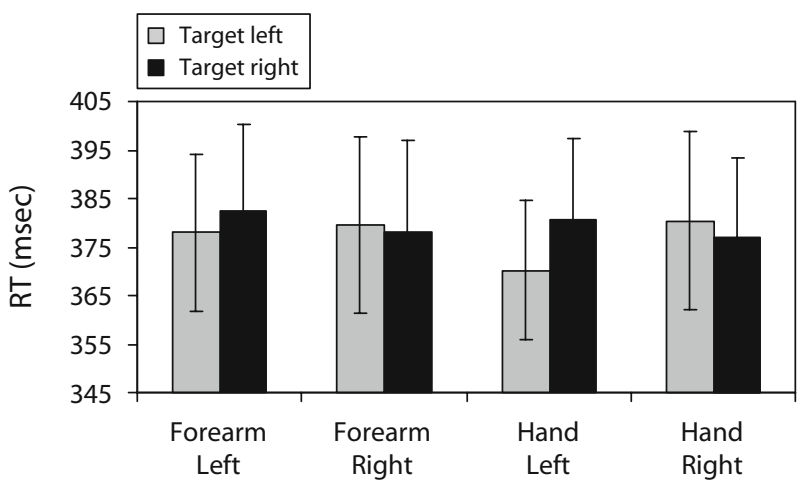

Part of Arm and Side

Figure 5. Results of Experiment 2. The results indicate a hand side $\times$ target side interaction, indicating facilitated response times (RTs) for targets appearing on the same side as the palm or forearm. Individual analyses of palm and forearm conditions revealed that this interaction was significant only in the palm condition. Error bars indicate $95 \%$ within-subjects confidence intervals.

condition did not $[F(1,28)=1.01$, n.s.]. A marginal effect was found for arm part and validity $[F(1,28)=3.83$, $\left.M S_{\mathrm{e}}=15,694.97, p=.06\right]$, suggesting a stronger validity effect in the hand condition than in the forearm condition. There were no other interactions $[F(1,28)<1]$. Thus, the bias was stronger for space near the hand than for that near the forearm. Again, these results indicate that our functional experiences in the world direct our attention to functionally relevant regions of space.

\section{EXPERIMENT 3}

\section{Functional Tool Use Extends Attention Bias to Extrapersonal Space}

In Experiments 1 and 2, we demonstrated that spatial attention can be biased by hand function and experience. The purpose of Experiment 3 was to investigate whether functional tool use and experience could extend the attention effect to space outside of the hand's normal reach. Iriki et al. (1996) reported that the receptive fields of handcentered bimodal cells in macaques expanded to represent space relative to a tool that the macaques held. Farné, Pavani, Menghello, \& Ládavas (2000) used cross-modal extinction to investigate whether visuotactile representations in humans were similarly malleable. They found that the visual stimuli presented near a tool that was held in the ipsilesional hand were as effective in causing contralesional tactile extinction as were stimuli presented near the ipsilesional hand itself. If, as these studies suggest, the representation of peripersonal space can be remapped to account for the effective extension of the arm by the tool, we should be able to induce the attention bias for stimuli presented near a tool with which participants have had functional experience. This bias should occur even if the length of the tool puts targets at distances that are outside of the hand's reach in extrapersonal space.

We compared hand and tool conditions. In the tool condition, the participants used a rake to manipulate sand in a Zen garden prior to testing and then held the rake to touch the computer screen. In the hand condition, the participants held their hand the same distance from the screen, but without the rake. If attention bias can be modified through functional experience, we would expect that the bias effect should be extended to the end of the rake even though it is outside of the participants' peripersonal space.

\section{Method}

Participants. Thirty-six right-handed undergraduates ( 27 female, mean age $=20.45$ years) from the University of Denver participated for extra credit in psychology courses.

Stimuli and Apparatus. The stimuli and visual displays were the same as those in the previous experiments. In addition, the participants used an $18-\mathrm{cm}$ wooden rake to make patterns in the sand of a rock-filled Zen garden $(20.32 \times 35.56 \mathrm{~cm})$.

Procedure. The procedure in Experiment 3 was identical to that in the previous experiments in terms of the covert-orienting paradigm, but hand conditions varied and participants practiced using a rake in a Zen garden. Tool conditions were compared with hand conditions. For the tool conditions, the participants held a small rake in one of their hands, with one end touching the computer screen, positioned next to the outer edge of the ipsilateral target box (see Figure 6). The rake was held with the prongs facing inward toward

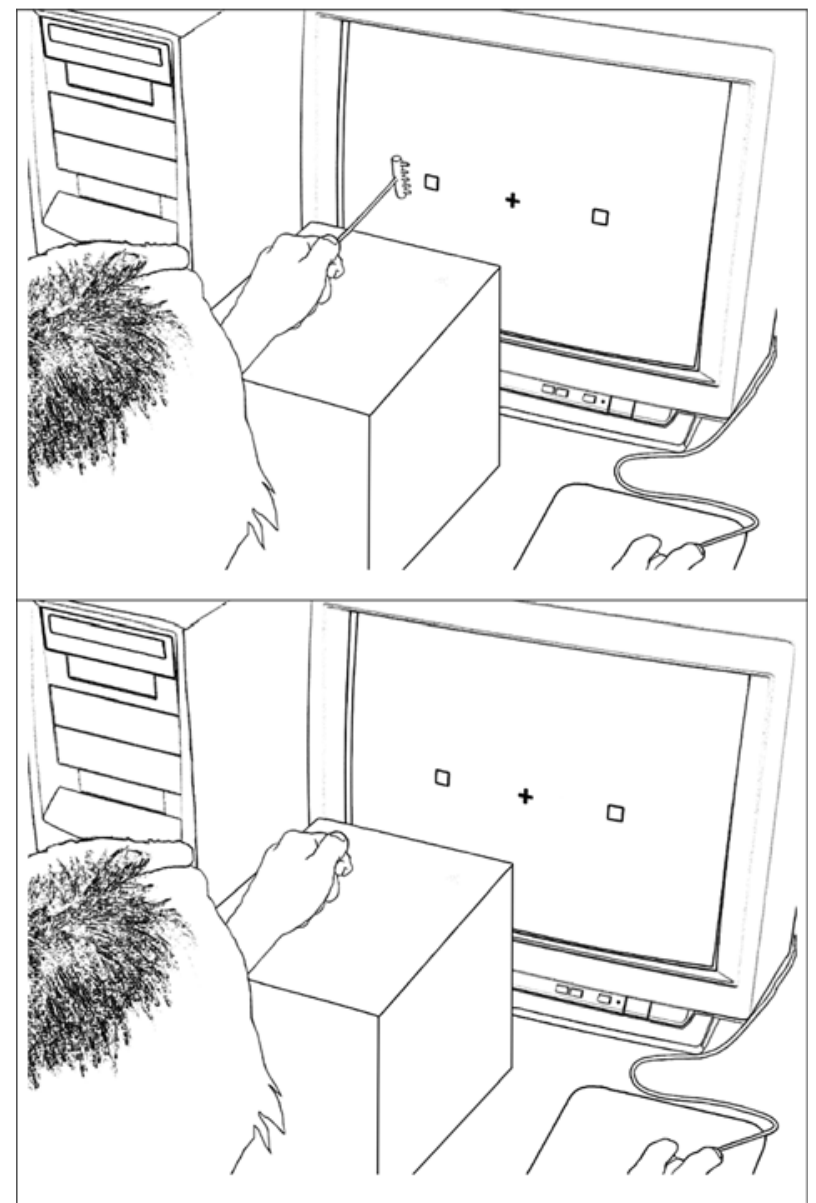

Figure 6. Experimental conditions for Experiment 3. Participants used a rake for $1 \mathrm{~min}$ prior to each block of tool trials. For the tool conditions, the rake was held so that the target box was in front of the rake in raking space. For the no-tool conditions, participants held their hand as if they held the rake (i.e., in the same grasping position and distance from the screen) but did not hold the rake. 
the target box and center of the screen. The participants held the rake with the hand grasping the rake $13 \mathrm{~cm}$ from the screen. Before testing in the tool conditions, the participants made sand designs of their own choice with the rake for $1 \mathrm{~min}$ in a Zen garden.

In the hand conditions, the participants did not use the rake in the Zen garden prior to experimental trials. They held their hand $13 \mathrm{~cm}$ away from the computer screen in a grasp posture, as if they were holding the rake outside the target box. In other words, the hand condition was similar to the rake condition, with the exception that the participants actually held the rake. There were two blocks of trials for each condition (i.e., tool left, tool right, hand left, and hand right), for a total of eight blocks of 50 trials each. Block order was randomized.

\section{Results and Discussion}

The data were processed in the same manner as in Experiment 1 . One participant responded in excess of $25 \%$ of the catch trials and was excluded from the analyses.

To determine whether functional experience using a tool could flexibly extend facilitation outside of peripersonal space, a $2 \times 2 \times 2 \times 2$ repeated measures ANOVA was conducted with factors of tool (rake, no rake), tool side (left, right), target side (left, right), and validity (valid, invalid). Confirming the basic paradigm, valid trials $(M=379.31$, $S E=10.19)$ were responded to more quickly than were invalid trials $(M=420.95, S E=13.15)$ [main effect of validity: $\left.F(1,34)=79.82, M S_{\mathrm{e}}=2,867.53, p<.0001\right]$. A tool side $\times$ target side interaction $\left[F(1,34)=5.99, M S_{\mathrm{e}}=\right.$ $541.56, p=.02]$ was mediated by the following predicted three-way interaction. Most important, the tool placed near a target position changed performance, but the hand held in the same position did not [see Figure 7; tool $\times$ tool side $\times$ target side interaction: $F(1,34)=4.13, M S_{\mathrm{e}}=646.36, p=$ $.050]$. To confirm the differential effects of tool use, separate tool side $\times$ target side $\times$ validity ANOVAs confirmed that the tool side $\times$ target side interaction occurred only for the rake condition $\left[F(1,34)=8.47, M S_{\mathrm{e}}=718.00, p=.006\right]$ and did not occur for the no-rake condition $[F(1,34)<1]$.

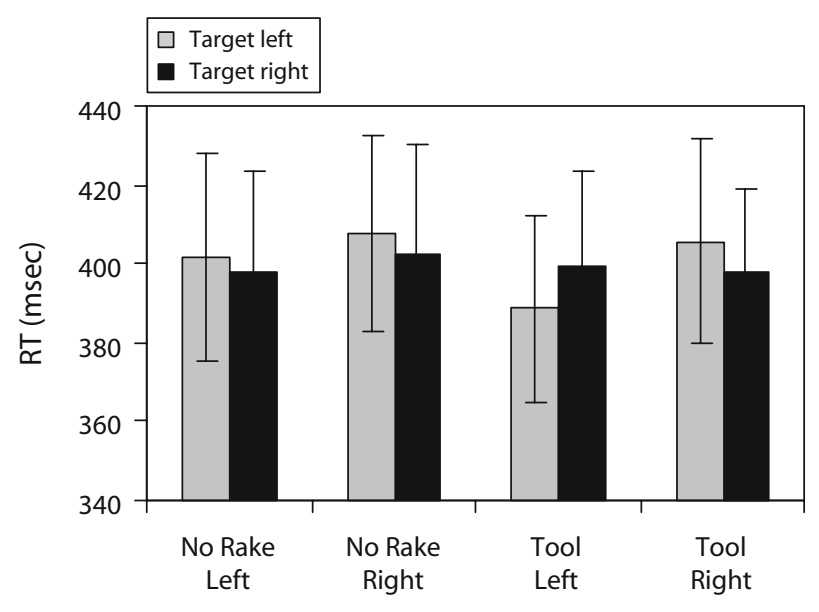

Tool Condition and Side

Figure 7. Results of Experiment 3. The results indicate a threeway interaction between tool use, hand side, and target side. They showed facilitated response times (RTs) only for targets appearing on the same side as the tool. Error bars indicate $95 \%$ withinsubjects confidence intervals.
This tool facilitation effect occurred for both validly and invalidly cued trials: The four-way interaction that included cue validity was not significant $[F(1,34)<1$, n.s.]. Tool use did not differentially influence the effect of visual cues (i.e., validity effect). No other main effect or interaction approached significance $(p>.09)$. In sum, the results of Experiment 3 suggest that the experience and functional tool use can bias attention outside of peripersonal space.

\section{EXPERIMENT 4 Topology of Attention Bias in Extended Peripersonal Space}

In Experiment 4, we investigated whether the topology of attention bias is altered through functional tool experience. This experiment replicated the design of Experiment 1, using the rake instead of the hand, in which the rake was positioned so that either the prong side or the back side was located next to the potential target locations. Before each block of trials, the participants were given a 1-min period of raking experience using the prongs of the rake to create patterns in the sand. If functional experience with the rake influences the distribution of attentional bias, as it does for the hand, relatively shorter RTs should be observed for targets appearing next to the prong side of the rake.

\section{Method}

Participants. Thirty-two undergraduates ( 22 female, mean age $=$ 20.70 years) from the University of Denver participated for extra credit in psychology courses.

Stimuli and Apparatus. The visual stimuli were identical to those used in the previous experiments. The rake and Zen garden were the same as those used in Experiment 3.

Procedure. The procedure was similar to that used in Experiment 1 , with the exception that the participants always held a rake up to the computer screen. The participants gained experience using the rake, following the same procedure as that described in Experiment 3. However, in this experiment, the rake was held in one of two positions. In the prong condition, the rake was held so that the prong side of the rake was held next to the outside line of the target box. In the back condition, the rake was held so that the back (i.e., flat side) of the rake was next to the inside line of the target box. For all the conditions, the participant sat $60 \mathrm{~cm}$ from the screen, and the arm was supported comfortably by a brace, with the shoulder relaxed. There were two blocks of trials for each condition (i.e., prong left, prong right, back left, and back right), for a total of eight blocks of 50 trials each. Block order was randomized.

\section{Results and Discussion}

The data were processed in the same manner as in Experiment 1 . One participant responded in excess of $25 \%$ of the catch trials and was excluded from the analyses.

To determine whether tool function affected the topology of attention bias in extrapersonal space, a $2 \times 2 \times 2 \times 2$ repeated measures ANOVA was conducted with factors of tool position (prong, back), hand side (left, right), target side (left, right), and validity (valid, invalid). Confirming the basic paradigm, valid trials $(M=359.16, S E=$ 8.69) were responded to more quickly than were invalid trials $(M=395.81, S E=13.56)$ [main effect of validity: $\left.F(1,30)=31.43, M S_{\mathrm{e}}=5,298.67, p<.0001\right]$. Most important, the way the tool was placed near a target posi- 


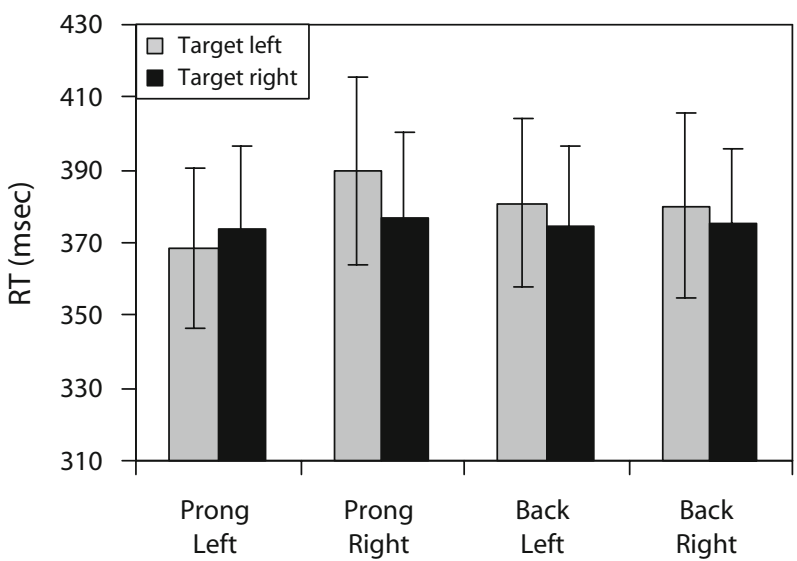

Rake Position and Rake Side

Figure 8. Results of Experiment 4. The results indicate a threeway interaction between tool position, hand side, and target side. They show facilitated response times (RTs) for targets appearing on the same side as the rake, but only when they appeared on the prong side of the rake. Error bars indicate $\mathbf{9 5 \%}$ within-subjects confidence intervals.

tion changed performance (i.e., tool position, with targets appearing to either the prong side of the rake or the back side of the rake). A tool position $\times$ hand side interaction $\left[F(1,30)=4.63, M S_{\mathrm{e}}=1,011.44, p<.040\right]$ was mediated by the three-way interaction. Targets appearing to the prong side of the rake were detected relatively more quickly than targets appearing to the back of the rake [see Figure 8; tool position $\times$ hand side $\times$ target side interaction: $\left.F(1,30)=4.37, M S_{\mathrm{e}}=693.96, p=.045\right]$. To confirm the differential effects of tool position, separate hand side $\times$ target side $\times$ validity ANOVAs were conducted for the prong and back conditions. The hand side $\times$ target side interaction occurred only in the prong condition $\left[F(1,30)=5.25, M S_{\mathrm{e}}=995.96, p=.029\right]$, not in the back condition $[F(1,30)<1$, n.s. $]$.

In addition, the four-way interaction that included cue validity was not significant $[F(1,30)<1$, n.s.]; the tool function bias effect occurred for both validly and invalidly cued trials. The functional tool space in which the target occurred did not differentially influence the effects of visual cues (i.e., the validity effect). No other main effect or interaction achieved significance.

In sum, targets appearing on the functional side of the rake (i.e., on the prong side of the rake) were detected more quickly than when they appeared at a location equidistant from the rake but on its back side. The tool's raking function appeared to facilitate the processing of targets appearing in "raking" space. These findings indicate that attention bias can be extended outside of peripersonal space via functional tool use. It is malleable to functional interactions with tools and the way that we use them.

\section{GENERAL DISCUSSION}

This study demonstrates that our functional interactions with the world shape our visual processing and empha- sizes the importance of incorporating bodily inputs into neurally based models of spatial attention. An embodied theory of spatial attention implies that our bodies and our experience using our bodies should help influence how attention is distributed in space and, as a result, how visual stimuli are processed (Reed et al., 2007). Recent work has demonstrated that the current locations of our body parts constrain our actions at any moment and influence where spatial attention is allocated (Grubb \& Reed, 2002; Reed et al., 2006): Targets appearing near the hand tend to be detected more quickly than targets appearing away from the hand in other regions of visual space. Here, we investigated the extent to which experience and hand function further constrain and define attentional allocation in perihand and peripersonal space. If attention helps prepare us for upcoming action, hand function and experience with tools should affect the topology of the attention bias around them.

Using a predictive visual covert-orienting paradigm similar to that in Reed et al. (2006), we compared the relative facilitation for targets appearing near different regions around the hand. Our results showed a relatively greater facilitation for targets appearing near the palm in grasping space versus near the back of the hand. Stronger facilitation was found for the space near the palm, relative to space near the forearm. These results suggest that attention bias is directed by the functional properties of the hand.

To confirm that the attention bias was related to functional interaction in visual space, we extended these findings to rake or tool use. We found that this same attention bias could be extended beyond the hand (and peripersonal space) to the end of a rake after participants had used the rake. In addition, an analogous functional topography of the bias was observed for targets appearing near prongs of the rake, relative to the back bar of the rake. The plasticity of attentional bias is more than a mere extension of hand extent, since it is also related to how the tool is used. Thus, the attentional facilitation of perihand/tool space appears to be related to the affordances presented by the presence of the hand or tool, as well as functional experience with the hand or tool.

Although we found consistent validity effects (i.e., faster responses to validly cued targets, relative to invalidly cued targets) across all conditions and in all four experiments, the presence and size of these validity effects did not interact with hand or tool location. This is important to note for two reasons. First, it indicates that hands and tools change the distribution of attention for the space in which targets appear because it is space in which those targets can be acted upon. The location of a passive hand or tool contributes to the control of attention, as does the peripheral cue, but their inputs do not seem to interact with each other. This is consistent with previous findings (Reed et al., 2006).

Second, the lack of interaction of the validity effect with hand or tool location in Experiments 2 and 4 refutes an argument that the hand or tool placed between the fixation and the target box might keep attention from being allocated past the hand or rake to the target boxes. Given that the same validity effect occurred regardless of hand 
or tool position, it appears that an intervening hand or tool does not affect the directing of attention. This provides further support that it is the functional properties of the hand or tool that produce the facilitation effect, rather than the location of the hand inhibiting attention.

The present findings might also be explained by eye movements. It is possible that the hand or tool acted as a prepotent stimulus. If so, the bias effect could be explained by participants' moving their eyes to locations near their hand or tool. To address this alternative explanation, we tested a subset of participants in Experiment 1, using a Tobii $120-\mathrm{Hz}$ eyetracker $(n=8$; Tobii Technology, Stockholm, Sweden). For each participant, fewer than $1 \%$ of the trials for each of the participants were invalidated for shifts in eye fixation. Thus, eye movements do not explain the facilitated performance.

The present results from these spatial attention tasks differ from those found from single-cell recordings in primates. The topology of attentionally biased space appears to have a different shape from primate bimodal receptive fields around the hand. Spatial attention emphasizes grasping space and functional tool space. In contrast, primate bimodal receptive fields appear to respond to visual stimuli in both grasping and avoidance space that is distributed more equally around the effector (e.g., Graziano \& Cooke, 2006; Graziano \& Gross, 1995). There are several reasons why the topology of the regions biased by attention in our experiments may differ from that in the primate data. First, we are measuring attentional effects, which are at a different level of analysis from the single-cell recordings of receptive fields. Second, there are some apparent experimental differences. In single-cell recording studies, it appears that the hand is held flat against the surface of the table. This hand orientation is different from that in our study, in which participants held their hand on the side with the thumb facing up. Thus, given the results of our study, hand orientation may influence the topology of attentional distribution. Last and perhaps most important, human experience in the world may be different from that of primates. In our controlled environments, humans may perform fewer avoidance behaviors and perform disproportionately more grasping behaviors to interact with objects. In contrast, monkeys in the lab and in the wild may perform many more swatting behaviors (e.g., to avoid other animals) and relatively fewer functional grasps with objects. Thus, attention bias may be more evenly distributed across both sides of the hand. In contrast, attention bias for the humans in our study was greater in grasping space.

This study confirms that the body and its functions are relevant to the allocation of spatial attention. How might these findings be integrated into a neurologically based model of spatial attention? The framework of the biased competition model (Desimone \& Duncan, 1995; Duncan, 2006) helps to explain the findings if multimodal contributions to visual attention are added to the basic model. In this model, inputs from multiple stimuli compete for representation in the visual cortex. Sensory inputs from the environment influence the competition from a bottomup direction. Top-down inputs from the frontoparietal network, including action goals and task demands, bias the competition in favor of relevant stimuli. The results of the present paradigm suggest that bimodal neurons may bias the competition for the side of space in which the hand or effector is located. When one hand is held in visual space, inputs from bimodal neurons that represent that location in space may combine with the visual stimuli to create an increase in attentional activation for the location of space in which the hand is located, thereby biasing the system for that location over other locations. Targets appearing near the hand may benefit from being relatively closer to a detection threshold than are targets in other parts of visual space. The locations near the hand, with their combined visual and bimodal spatial representations, alter the resting distribution of spatial attention.

Functional experience can influence this competition in two ways. First, it can provide top-down inputs with changes in expectations regarding where relevant stimuli are likely to appear. Second, it can alter the distribution of attention in perihand space, potentially arising from changes in the receptive field properties of the bimodal neurons. With experience using the hand or the tool, the underlying spatial representation from the bimodal neurons can be changed to reflect the optimization of action.

The influence of functional experience suggests that affordances or hand-object interactions may also bias attention not only for regions of space near the hand, but also for specific regions of space between the hand held in a particular posture and the corresponding shape of the object to be used. If the hand is held in the posture in which one typically uses the object, there may be attentional effects in the form of hand-object affordances.

In conclusion, this study provides a novel addition to the biased competition model of attention. Previous models do not account for the important role that the body and its functions play in directing spatial attention. A primary function of spatial attention is to plan physical actions, and these actions are based on the current locations and functions of our hands and tools. Together, our experiments suggest that the processing of targets in functional action space is facilitated to help us prepare effective responses to visual events. Nonetheless, in these experiments, the hand and tools are held in a static posture. Future research is needed to examine how the actual actions of the hand influence the dynamics of spatial attention.

\section{AUTHOR NOTE}

The authors thank Jeff Grubb for his insights and technical contributions. In addition, we thank Anna Vazquez, Nashwa Bolling, Carolyn Nystrom, Josh Baker, George Park, and Casey Wieland for their help in data collection. Correspondence concerning this article should be addressed to C. L. Reed, Department of Psychology, Claremont McKenna College, 850 Columbia Ave., Claremont, CA 91711 (e-mail: clreed@) cmc.edu).

\section{REFERENCES}

Berti, A., \& Frassinetti, F. (2000). When far becomes near: Remapping of space by tool use. Journal of Cognitive Neuroscience, 12, 415-420.

Braun, J., Koch, C., \& Davis, J. L. (2001). Visual attention and cortical circuits. Cambridge, MA: MIT Press.

Cooke, D. F., \& Graziano, M. S. A. (2004). Sensorimotor integration in the precentral gyrus: Polysensory neurons and defensive movements. Journal of Neurophysiology, 91, 1648-1660. 
Desimone, R., \& Duncan, J. (1995). Neural mechanisms of selective visual attention. Annual Review of Neuroscience, 18, 193-222.

Duncan, J. (2006). Brain mechanisms of attention. Quarterly Journal of Experimental Psychology, 59, 2-27.

FARNÉ, A., \& LÁDAVAS, E. (2000). Dynamic size-change of hand peripersonal space following tool use. NeuroReport, 11, 1645-1649.

Farné, A., Pavani, F., Menghello, F., \& Ládavas, E. (2000). Left tactile extinction following visual stimulation of a rubber hand. Brain, 123, 2350-2360.

GraZiano, M. S. A. (2001). A system of multimodal areas in the primate brain. Neuron, 29, 4-6.

Graziano, M. S. A., \& Cooke, D. F. (2006). Parieto-frontal interactions, personal space, and defensive behavior. Neuropsychologia, 44, 845-859.

Graziano, M. S. A., \& Gross, C. G. (1994). Mapping space with neurons. Current Directions in Psychological Science, 3, 164-167.

Graziano, M. S. A., \& Gross, C. G. (1995). The representation of extrapersonal space: A possible role for bimodal visual-tactile neurons. In M. S. Gazzaniga (Ed.), The cognitive neurosciences (pp. 10211034). Cambridge, MA: MIT Press.

Graziano, M. S. A., Taylor, C. S. R., Cooke, D. F., \& Moore, T. (2005). A map of complex movements in motor cortex of primates. In G. W. Humphreys \& M. J. Riddoch, Attention in action: Advances in cognitive neuroscience (pp. 211-232). London: Taylor \& Francis, Psychology Press.

GrubB, J. D., \& ReED, C. L. (2002). Trunk orientation induces neglect-like lateral biases in covert attention. Psychological Science, 13, 553-556.

Grubb, J. D., Reed, C. L., Bate, S., Garza, J., \& Roberts, R. J., JR. (2008). Walking reveals trunk orientation bias for visual attention. Perception \& Psychophysics, 70, 688-696.

Hasselbach-Heitzeg, M. M., \& Reuter-Lorenz, P. A. (2002). Egocentric body-centered coordinates modulate visuomotor performance. Neuropsychologia, 40, 1822-1833.

Holmes, N. P., Calvert, G. A., \& Spence, C. (2006). Tool use changes multisensory interactions in seconds: Evidence from the crossmodal congruency. Experimental Brain Research, 183, 465-476.

Iriki, A., Tanaka, M., \& Iwamura, Y. (1996). Coding of modified body schema during tool use by macaque postcentral neurons. NeuroReport, 7, 2325-2330.
Ládavas, E., Pellegrino, G., Farné, A., \& Zeloni, G. (1998). Neuropsychological evidence of an integrated visuotactile representation of peripersonal space in humans. Journal of Cognitive Neuroscience, 10, 581-589.

Maravita, A., Spence, C., Kennett, S., \& Driver, J. (2002). Tool-use changes multimodal spatial interactions between vision and touch in normal humans. Cognition, 83, B25-B34.

Pashler, H. (1998). Attention. San Diego: Psychology Press.

Posner, M. I., \& Cohen, Y. (1984). Components of visual orienting. In H. Bouma \& D. G. Bouwhuis (Eds.), Attention and performance X: Control of language processes (pp. 531-555). Hillsdale, NJ: Erlbaum.

Posner, M. I., Walker, J. A., Friedrich, F. J., \& Rafal, R. D. (1987). How do the parietal lobes direct covert attention? Neuropsychologia, 25, 135-146.

Previc, F. H. (1998). The neuropsychology of 3-D space. Psychological Bulletin, 124, 123-164.

Reed, C. L., GarZa, J. P., \& Roberts, R. J., JR. (2007). The influence of the body and its actions on spatial attention. In L. Paletta \& E. Rome (Eds.), Attention in cognitive systems (pp. 42-58). Berlin: Springer.

Reed, C. L., Grubb, J. D., \& Steele, C. (2006). Grasping attention: Behavioral consequences of bimodal neurons. Journal of Experimental Psychology: Human Perception \& Performance, 32, 166-177.

Rizzolatti, G., Gentilucci, M., \& Matelli, M. (1985). Selective spatial attention: One center, one circuit, or many circuits? In M. I. Posner \& O. S. Marin (Eds.), Attention and performance XI (pp. 251265). Hillsdale, NJ: Erlbaum.

TipPer, S. P. (2004). Attention and action. In M. Gazzaniga (Ed.), The cognitive neurosciences (3rd ed., pp. 619-630). Cambridge, MA: MIT Press.

Vaishnavi, S., Calhoun, J., \& Chatterjee, A. (1999). Crossmodal and sensorimotor integration in tactile awareness. Neurology, 53, 1596-1598.

VeCERA, S. P., \& Rizzo, M. (2003). Spatial attention: Normal processes and their breakdown. Neurologic Clinics of North America, 21, 575607.

(Manuscript received February 2, 2009; revision accepted for publication August 19, 2009.) 